\title{
Lateral Migration and Nonuniform Rotation of Square Particle Suspended in Poiseuille Flow
}

\author{
Yong Ye1,2, Huajie Zhou1,2, Sihao Zhou1,2, Zhangrong Qin ${ }^{1,2}$, Binghai Wen ${ }^{1,2}$ \\ ${ }^{1}$ Guangxi Key Lab of Multi-Source Information Mining \& Security, Guangxi Normal University, Guilin, China \\ ${ }^{2}$ Department of Computer Science and Information Engineering, Guangxi Normal University, Guilin, China \\ Email: oceanwen@gxnu.edu.cn, qinzhangrong@gxnu.edu.cn
}

How to cite this paper: Ye, Y., Zhou, H.J., Zhou, S.H., Qin, Z.R. and Wen, B.H. (2020) Lateral Migration and Nonuniform Rotation of Square Particle Suspended in Poiseuille Flow. Journal of Flow Control, Measurement \& Visualization, 8, 146-158. https://doi.org/10.4236/jfcmv.2020.83009

Received: December 2, 2019

Accepted: June 23, 2020

Published: July 29, 2020

Copyright $\odot 2020$ by author(s) and Scientific Research Publishing Inc. This work is licensed under the Creative Commons Attribution International License (CC BY 4.0).

http://creativecommons.org/licenses/by/4.0/

\begin{abstract}
A square particle suspended in a Poiseuille flow is investigated by using the lattice Boltzmann method with the Galilean-invariant momentum exchange method. The lateral migration of Segré-Silberberg effect is observed for the square particle, accompanied by the nonuniform rotation and regular wave. To compare with the circular particle, its circumscribed and inscribed squares are used in the simulations. Because the circumscribed square takes up a greater difference between the upper and lower flow rates, it reaches the equilibrium position earlier than the inscribed one. The trajectories of the latter are much closer to those of circle; this indicates that the circle and its inscribed square have a similar hydrodynamic radius in a Poiseuille flow. The equilibrium positions of the square particles change with Reynolds number and show a shape of saddle, whereas those of the circular particles are virtually not affected by Reynolds number. The regular wave and nonuniform rotation are owing to the interactions of the square shape and the parabolic velocity distribution of Poiseuille flow, and high Reynolds number makes the square rotating faster and decrease its oscillating amplitude. A series of contours illustrate the dynamic flow fields when the square particle has successive postures in a half rotating period. This study is beneficial to understand the motion of anisotropic particles and the dendrite growth in dynamic environment.
\end{abstract}

\section{Keywords}

Particle Suspension, Square Particle, Segré-Silberberg Effect, Lattice Boltzmann Method

\section{Introduction}

The cross-flow phenomenon [1] caused by inertia of suspended particles in the 
Poiseuille flow has received extensive attention and research since Segré and Silberberg reported that neutrally buoyant spheres in a Poiseuille flow would laterally migrate to a certain equilibrium position between the pipe wall and the centerline; that phenomenon was named as the Segré-Silberberg effect [2]. In the 1960s, Karnis et al. [3] performed a lot of experiments to simulate various kinds of particles suspended in a three-dimensional channel and verified that the lateral migration is due to inertial effect. Recently, Matas et al. [4] examined the influence of inertia on the radial migration of rigid spherical particles in Poiseuille flow, and observed an accumulation of particles on an inner annulus, i.e. at smaller radial position than the Segré-Silberberg annulus. Huang et al. [5] investigated the sedimentation of an ellipsoid in both narrow circular and square tubes, also observed several modes of motion that are all independent of the initial orientation and found that a lighter ellipsoid may settle faster than a heavier one. The cross-stream migration is also used in the inertial microfluidics to focus and separate the suspended particles [6]. The curved channels are utilized to accelerate the migration of the particles [7] [8]. With the development of computer technology, many researchers implemented a lot of numerical simulations on particle migrations. Especially, the lattice Boltzmann method (LBM), as a credible computational hydrodynamics method, has achieved great progress in recent years [9]. The inherent advantages of simple algorithm, easy implementation of complex boundaries, high efficiency and full parallelization enable its successful applications on modeling complex fluid systems [10]. Sun et al. [11] studied the particle focusing in three-dimensional rectangular channel with the lattice Boltzmann method. Wen et al. [12] [13] simulated the movement of red blood cells of birds by studying the sedimentation migration of elliptical particles in shear flow [14] and Poiseuille flow, and positively contributed to the study of blood circulation of birds with elliptical red blood cells. Polygonal shape geometric boundary did not obtain sufficient investigations, although the suspension motion of these shaped particles widely exists in nature and many applications in industries, such as chemical, biological, and mechanical engineering [15]. To understand the movements of some suspended particles with square, triangle, or trapezoid shape [16], it is meaningful to construct an effective model to accurately simulate their behaviors.

This paper is organized as follows. In Section 2, we will list the numerical method we employ to simulate particle suspension in Poiseuille flow. The comparison results of suspension particles are performed in several aspects, which include migration trajectories, the impact of Reynolds number to equilibrium position and rotation, and are revealed in Section 3. Finally in Section 4, the summary of simulation and outlook will be described.

\section{Numerical Method}

\subsection{Lattice Boltzmann Method}

In this paper, we intend to investigate motion of a square particle suspended in a 
two-dimensional Poiseuille flow by using a single relaxation time lattice Boltzmann model. In single relaxation time lattice Boltzmann model, the distribution functions obey the following evolvement equation [17] [18] [19]

$$
f_{i}\left(\boldsymbol{x}+\boldsymbol{e}_{i} \delta t, t+\delta t\right)-f_{i}(\boldsymbol{x}, t)=-\frac{1}{\tau}\left[f_{i}(\boldsymbol{x}, t)-f_{i}^{(e q)}(\boldsymbol{x}, t)\right]
$$

where $f_{i}(x, t)$ is the distribution function at site $x$ and time $t, \tau$ and $\delta t$ denote the relaxation time and time step respectively, and $f_{i}^{(e q)}(x, t)$ is the equilibrium distribution function, which takes the form

$$
f_{i}^{(e q)}(\boldsymbol{x}, t)=\rho \omega_{i}\left[1+3\left(\boldsymbol{e}_{i} \cdot \boldsymbol{u}\right)+\frac{9}{2}\left(\boldsymbol{e}_{i} \cdot \boldsymbol{u}\right)^{2}-\frac{3}{2} \boldsymbol{u}^{2}\right]
$$

where weight factors $\omega_{i}$ are given by $\omega_{0}=4 / 9, \omega_{1-4}=1 / 9, \omega_{5-8}=1 / 36$ [20], $\rho$ and $\boldsymbol{u}$ are the macroscopic density and the macroscopic velocity vector respectively, are given by

$$
\rho=\sum_{i} f_{i} \text { and } \boldsymbol{u}=\frac{1}{\rho} \sum_{i} \boldsymbol{e}_{i} f_{i}
$$

During a time step, the particle distribution functions in a lattice site collide and then flow into its neighboring lattice sites [21]. The discrete velocities and equilibrium distribution functions depend on the particular velocity model. For a D2Q9 (two-dimensional, nine velocity) model, $\boldsymbol{e}_{i}$ is the discrete velocity along the $i t h$ lattice direction, and defined as $\{(0,0),(1,0),(0,1),(-1,0),(0,-1)$, $(1,1),(-1,1),(-1,-1),(1,-1)\}$ in nine directions respectively.

\subsection{Moving Boundary Conditions and Hydrodynamic Force Evaluation}

The LBM can effectively simulate the fluid flow in complex flow fields. However, the reliability of the simulation results strongly depends on the selected boundary treatment methods [22] [23]. In this paper a second-order interpolation boundary condition proposed by Lallemand et al. [20] is employed to treat the boundaries of the tube and particle, which is given by

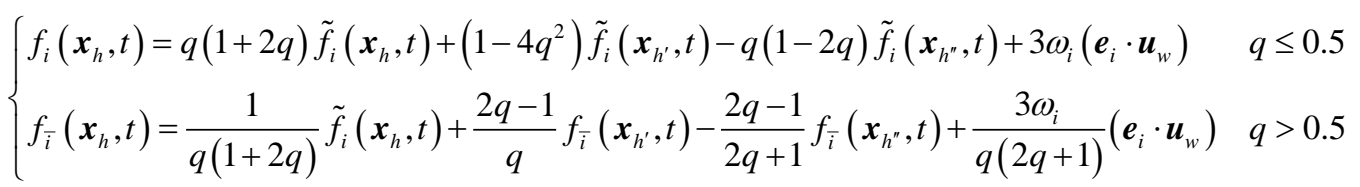

where the parameter $q$ defines the fraction in fluid region of a grid spacing intersected by the boundary, $\tilde{f}_{i}\left(x_{h}, t\right)$ is the distribution function streamed from $\boldsymbol{x}_{h}, \quad \boldsymbol{x}_{h^{\prime}}$ and $\boldsymbol{x}_{h^{\prime \prime}}$ are two points adjacent to $\boldsymbol{x}_{h}$ along $i$ direction, $f_{\bar{i}}$ is the distribution function of the reverse of $\boldsymbol{e}_{i}$ vector, $\boldsymbol{u}_{w}$ represents the velocity of the moving boundary, and $\omega_{i}$ takes $2 / 9$ for $i$ equals 1 to 4 and takes $1 / 18$ for $i$ equals 5 to 8 , respectively [21] [22] [23] [24].

The hydrodynamic force can be simply and efficiently evaluated by using a momentum exchange method. Ladd et al. [25] proposed a momentum exchange method which regards the solid lattices inside of a solid particle as the fluid lat- 
tices. Aidun et al. [26] improved the method to evaluate hydrodynamic force by using a half-way bounce condition, in which the particle is identified impervious. Wen et al. [12] [13] [20] introduced the relative velocity into the interfacial momentum transfer to compute the hydrodynamic force and proposed a Galilean invariant momentum (GME) exchange equation

$$
\boldsymbol{F}\left(\boldsymbol{x}_{s}\right)=\left(\boldsymbol{e}_{i}-\boldsymbol{v}\right) f_{i}\left(\boldsymbol{x}_{f}, t\right)-\left(\boldsymbol{e}_{\bar{i}}-\boldsymbol{v}\right) f_{\bar{i}}\left(\boldsymbol{x}_{b}, t\right)
$$

where $\boldsymbol{x}_{f}$ and $\boldsymbol{x}_{b}$ are the position of the fluid node and solid node which are lying on fluid-solid link both ends, and $\boldsymbol{x}_{s}$ is the intersection of the fluid-solid link with the solid particle boundary, $v$ is the velocity of the moving boundary [12] [27] [28]. The total hydrodynamic force and torque are calculated by

$$
\boldsymbol{F}=\sum \boldsymbol{F}\left(\boldsymbol{x}_{s}\right) \text { and } \boldsymbol{T}=\sum\left(\boldsymbol{x}_{s}-\boldsymbol{R}\right) \times \boldsymbol{F}\left(\boldsymbol{x}_{s}\right)
$$

where $\boldsymbol{F}$ and $\boldsymbol{T}$ are the summation of force and torque on each $\boldsymbol{x}_{s}, \boldsymbol{R}$ is the mass center of the solid particle. GME is simple, efficient in computation, and clearly expressed physically. Wen et al. used GME to compare the three-dimensional sphere migration experiments in the pipeline that made by Segré-Silberberg in the 1960s [29].

\section{Verifications of the Numerical Method}

To verify the numerical method, two simulations of cylinder sedimentations in a vertical channel were performed; one is a circular cylinder and is compared with the results of the arbitrary Lagrangian-Eulerian technique (ALE), the other is an elliptical cylinder and is compared with the results of the finite-element method (FEM). In the simulations, the gravity takes $|\boldsymbol{G}|=9.8 \mathrm{~m} / \mathrm{s}^{2}$ and the kinematic viscosity is $1 \times 10^{-6} \mathrm{~m}^{2} / \mathrm{s}$. The fluid field was designed as a vertical channel, and the width is $4 \mathrm{~mm}$.

In the first case, the width and height of the channel take 120 and 1200 lattice units, and the fluid density is $1 \times 10^{3} \mathrm{~kg} / \mathrm{m}^{3}$, while the density of the elliptical particle is $1.03 \times 10^{3} \mathrm{~kg} / \mathrm{m}^{3}$. The Reynolds number of circle particle is 8.33 , which is defined as $\operatorname{Re}=H U / v$, where $U$ denotes the final velocity of the particle and $v$ is the kinematic viscosity. The cylinder diameter is $1 \mathrm{~mm}$, and was released at $0.76 \mathrm{~mm}$ away from left wall. The results of GME shown in Figure 1 are always in excellent agreement with the benchmarks from the arbitrary Lagrangian-Eulerian technique (ALE) [30]. The above numerical results demonstrate the reliability and stability of GME when simulating particles motion with geometric symmetry.

For furtherly investigating the motion nature of geometrically asymmetric particles, the sedimentations of elliptical cylinder were simulated. In the second case, the major and minor axes of the ellipse are $a$ and $b$, and the width of the channel is $L$. Two dimensionless parameters are defined as aspect ratio $\alpha=b / a$ and blockage ratio $\beta=a / L$. The fluid density is $1 \times 10^{3} \mathrm{~kg} / \mathrm{m}^{3}$, while the density of the elliptical particle is $1.1 \times 10^{3} \mathrm{~kg} / \mathrm{m}^{3}$. The width and height of the channel take 120 and 1000 lattice units. Figure 2 shows the trajectories of elliptical cyl- 
inder sedimentations, two elliptical particles that are different in size are released at the middle of channel on horizontal direction with an initial angle of $\theta=\pi / 4$ and $\theta=\pi / 3$, respectively. The major and minor axes of the ellipse where in Figure 2 (a) are $1 \mathrm{~mm}$ and $0.5 \mathrm{~mm}$, and are $2.6 \mathrm{~mm}$ and $1.3 \mathrm{~mm}$ where in Figure 2(b). It is evident that the results are in accordance with the benchmarks from the finite-element method (FEM) [31] [32].

\section{Comparison Results from Suspension of Particles}

Figure 3 depicts a square particle suspended in a Poiseuille flow. A circular particle is used as a reference comparing with its circumscribed and inscribed square particles; thus, between square particles the side length of the former is equal to the length of the diagonal lines of the latter, and both are equal to the circular diameter. The width of the channel is $1 \mathrm{~mm}$ and the diameter of the circular particle is $0.25 \mathrm{~mm}$. The fluid density is $1 \times 10^{3} \mathrm{~kg} / \mathrm{m}^{3}$ and the kinematic viscosity coefficient is $v=1 \times 10^{-6} \mathrm{~m}^{2} / \mathrm{s}$, the density of particles is equal to the fluid. In the present simulations, the width $H$ of the channel is 120 lattice units ( $1 \mathrm{~mm}=120$ lattices), while the length $L$ takes 10 times the width; and then the diameter of the circle is 30 lattice units. Reynolds number $(R e)$ is a dimensionless number to characterizes fluid flow and is expressed as $R e=H U / v$, where $U$ is the mean fluid velocity in Poiseuille flow without a particle.

The pressure boundary condition is applied at inlet and outlet of the channel, while the second-order interpolation boundary condition is implemented for the boundaries of particles and the channel wall [33] [34]. The particles are placed initially at the middle of the channel in the horizontal direction and are released at 25 and 45 lattice units in the vertical direction. Please note that clockwise is defined as the positive direction to investigate the rotation of the particle.
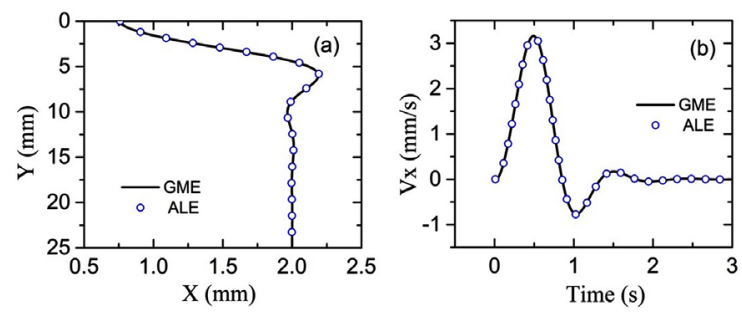

Figure 1. (a) Trajectories, (b) horizontal velocities of cylinder relative to the channel in sedimentations.
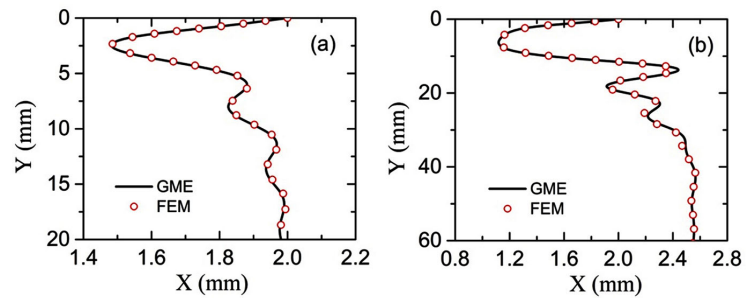

Figure 2. Trajectories of elliptical cylinder sedimentations. The dimensionless parameters are $\alpha=0.5$ and $\beta=0.25$ in (a), and are $\alpha=0.5$ and $\beta=0.6$ in (b). 


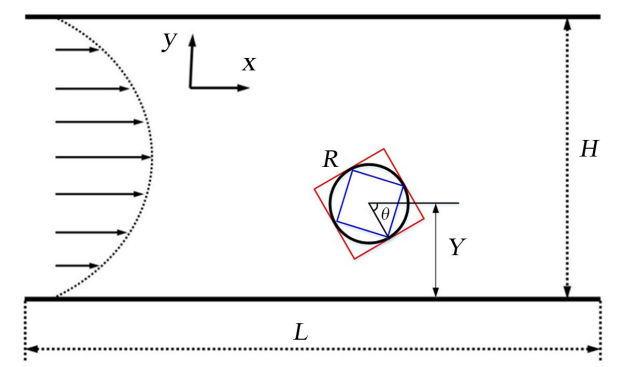

Figure 3. Schematic diagram of a particle suspended in Poiseuille flow. A circular particle (in black) is compared with its circumscribed (in red) and inscribed (in blue) square particles.

Figure 4 manifests the migration trajectories of the three types of particles. The black curve represents the trajectory of the circular particle, namely the classical Segré-Silberberg effect, while the red and blue curves represent the circumscriber's and inscriber's, respectively. It is obvious that the trajectories of the square particles include periodic waves owing to their noncircular geometries. Especially, the two trajectories and the equilibrium position of the inscribed particle are quite close to those of the circle. This indicates that the circle has a similar hydrodynamic radius to its inscribed square, although they are differences in mass. The trajectories of the circumscribed particle have larger oscillations; its equilibrium is closer to the channel centerline and it is faster to reach the equilibrium state than the inscribed one. These are due to the larger hydrodynamic radius and $R e$ of the circumscribed particle. The results are in consistent with the previous researches for circular and elliptical particles [11] [35]. The mass of the inscribed square is smaller than the circular, the uneven distribution of the flow velocity between upper and lower part in the channel pushes the particle to the equilibrium position. Due to its square geometry, it is sensitively affected by the uneven flow velocity, consequently the equilibrium position of the inscribed particle is slightly higher than the circular one. However, the mass of the circumscribed particle is significantly larger than the circular one; its equilibrium position is also much higher than it. On the time of the particles reaching equilibrium position, the large circumscribed particle takes up a greater difference between the upper and lower flow rate, and produces a larger thrust to make it to reach the equilibrium position earlier than the inscribed one.

The velocity of the fluid influences the movement of a square particle strongly. A series of equilibrium positions and rotation periods of the square under various $R e$ are shown in Figure 5. We found that the trajectory of equilibrium position of the two types of square particles just looks like a saddle, while what for the circular particles is less affected by the change of $R e$. Due to the Segré-Silberberg effect, the solid particles are subjected to both viscous drag force and inertial lift force. The drag force is responsible for driving particles along the flow streamlines, however, the inertial lift force results in the lateral migration of particles across the flow streamlines [26]. With the increase of $R e$, 


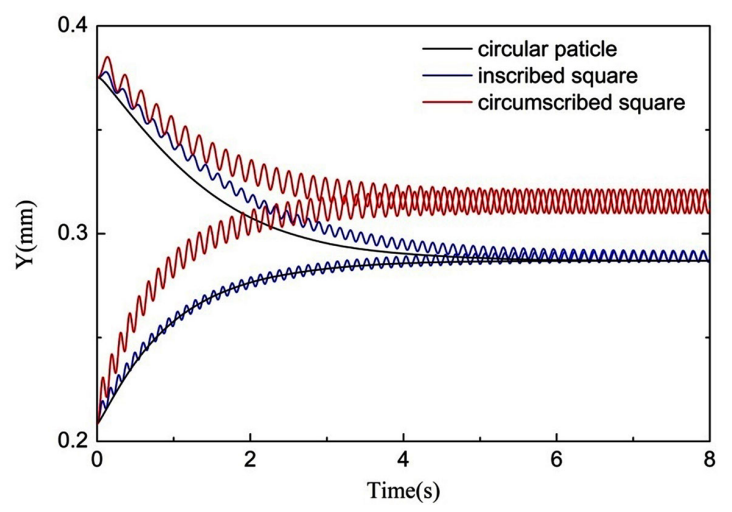

Figure 4. The comparison among circular particle and two types of square particle in $R e=10$. The origin of the $\mathrm{Y}$ axis is the lower boundary of the entrance to the pipeline, and this method applies to all pictures below.

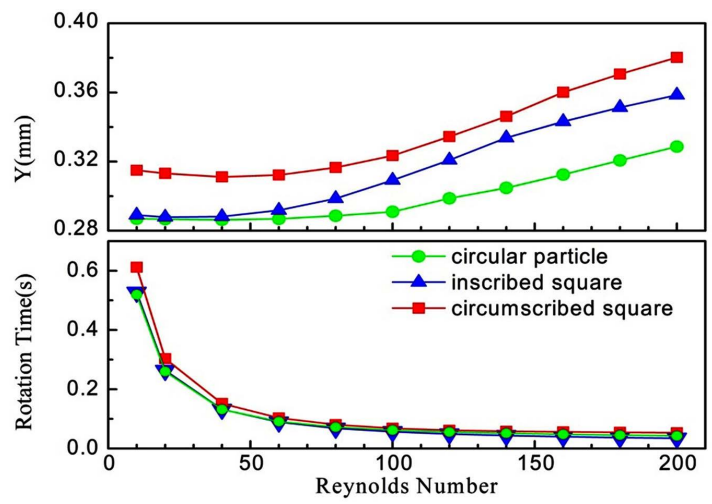

Figure 5. The effect of $R e$ on the final equilibrium position and particle rotation period. The red, blue and green line represents the circumscribed, the inscribed and the circular particle, respectively.

the inertial force grows gradually and then leads to a faster lateral migration. When $R e$ is higher than 100, the equilibrium positions of the circle and square particles appear to linearly increase towards the centerline. The particle would rotate more quickly, the rotation period decreases exponentially. The difference in the rotation period among the circumscribed, the inscribed and circular one is significantly reduced simultaneously.

The $R e$ reflects the intensity of the interaction between inertial and viscous forces. In order to observe the impact of motion by $R e$, five circumscribed square particles are released at lower position which relative to half flow filed, and their final equilibrium positions and the trajectories are shown in Figure 6. When $R e$ is small, such as 10 , the fluctuation is highest among the trajectories of square particles, but is getting smaller with the growth of $R e$. The equilibrium positions of the square particles which correspond to the saddle curves illustrated in Figure 5 tend to move to the lower wall when Re takes from 10 to 40, but move to the upper wall if $R e$ takes 40 to 200. 


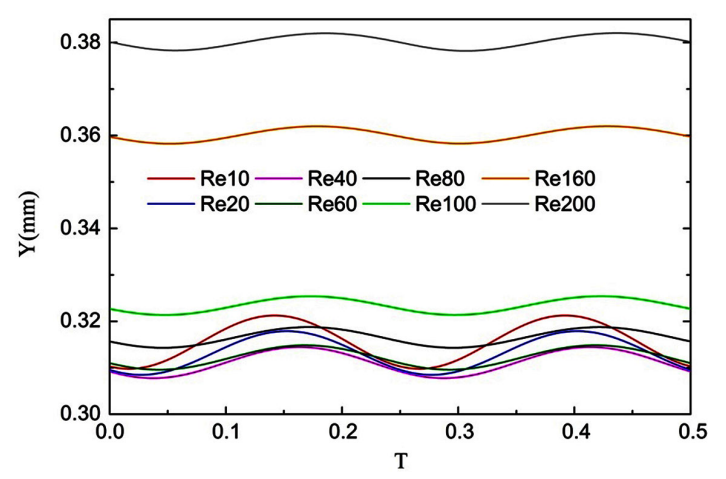

Figure 6. The influence of different $R e$ to the trajectory of square particles in a half rotation cycle. A complete period is defined as that a process of 360 degrees of rotation orientation angle of particles after reaching a stable equilibrium position.

As the circular particle has an axisymmetric geometry, its migration trajectory does not have additional fluctuations and nonuniform rotation. In contrast to the circular particles, the migration trajectories of the square ones are quite similar to the classical Segré-Silberberg effect, although their unsymmetrical geometries produce additional fluctuations and nonuniform rotation in the process of migration. The extra fluctuations and nonuniform rotations in the simulations are due to the interaction of the shape of the particle and the parabolic velocity distribution in channel. The fluid velocity close to centerline is invariably faster than the farther one and the speed difference drives the particles to rotate continuously [12]. Figure 7 shows the steering angle, vertical trajectory, angular velocity of the circumscribed square particles, and the equilibrium position corresponding to the steering angle in a half rotation period. The particle equilibrium position reaches the peaks when the steering angle is around $0.3 \pi$ and $0.8 \pi$. The maximum and minimum angular velocity are both in the middle of the wave peak and the trough. The alternating hydraulic force caused by the particle rotation becomes a lift force in the angular range of $0.05 \pi-0.3 \pi$, and a falling force at $0.3 \pi-0.55 \pi$.

Poiseuille flow is a typical laminar flow. The vertical velocity produced by the particulate fluctuation and rotation is more sensitive than the horizontal velocity. Some contour plots are shown in Figure 8. A square particle is released in the fluid filed which is 1800 lattices long and 180 lattices width, and $R e$ is 250 . Specially, since the length unit is based on mesh in this simulation, unit of time, length, velocity, viscosity coefficient and so on are dimensionless. The steering angles are $0, \pi / 12, \pi / 6, \pi / 4, \pi / 3$, and $5 \pi / 12$, corresponding to Figures $8(\mathrm{a})$-(f), respectively. The particle is at 0 degree, and it can be illustrated that the surrounding flow velocity distribution is even and gentle in the subfigure (a). The subfigures (b)-(d) illustrate the rising process of a square particle due to the effect of lift force. The subfigures (e) and (f) depict the falling process of square particle under the falling force dominates the flow field. 


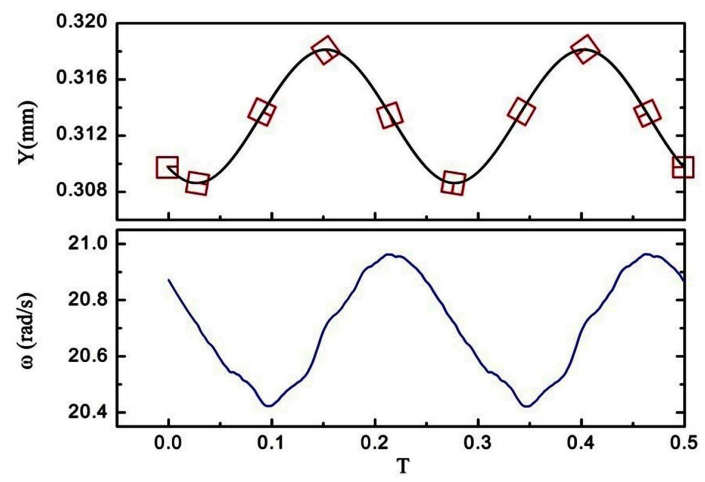

Figure 7. The trajectory and the angle velocity of the square particle in the equilibrium state in a half rotation period, together with the particle orientations.

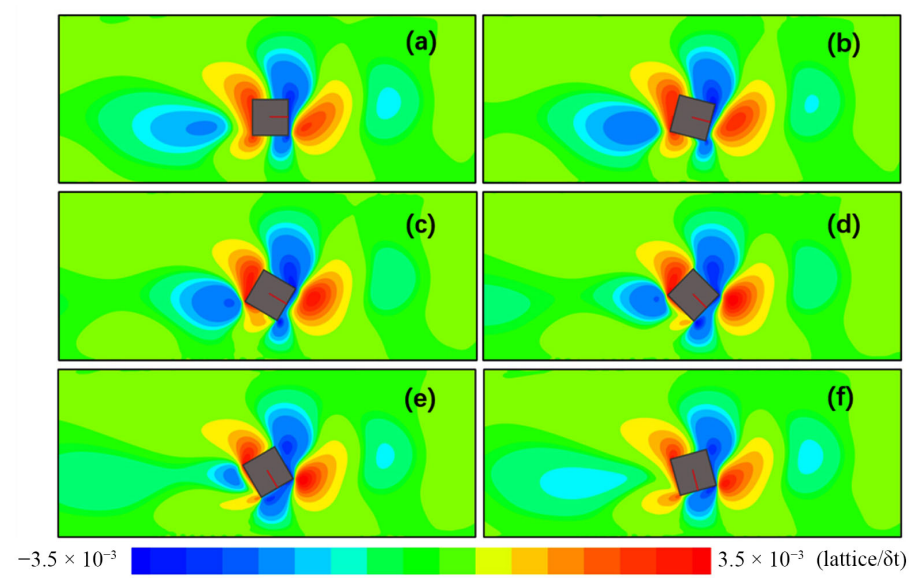

Figure 8. The contours of the fluid flow around the square particle with various steering angles. The speed unit defined as the lattice per step time.

\section{Summary and Outlook}

In summary, a square particle suspended in a Poiseuille flow is numerically investigated and compared with a circular particle by using the lattice Boltzmann method. The hydrodynamic force is evaluated by the Galilean-invariant momentum exchange method. The lateral migration and equilibrium of the square particle are consistent with the classical Segré-Silberberg effect. Besides, the movement of the square particle includes nonuniform rotation and regular fluctuation due to its noncircular geometry. Comparing the circular particle with its inscribed and circumscribed square particles, the equilibrium position of the circular particles remains almost unchanged with different $R e$, while the square particles show that a saddle-shaped position distribution and their rotation period decrease exponentially with the increase of $R e$. Furthermore, the fluid in high $R e$ pushes the square rotating faster and restrains its oscillating amplitude. The contour of the velocity vividly shows the changes in the flow field around the particles. Due to its advantages in calculating particles with complex shapes, meanwhile the calculation process of GME does not interfere with the physical 
parameters in the flow field and it can be directly extended to 3D simulations. Considering the extension in $3 \mathrm{D}$, the computation complexity in calculating geometry on solid surfaces will be increased complexity by an order of magnitude. Researchers should pay more attention to the construction of the particle geometry and the processing of flow field boundary in $3 \mathrm{D}$, in order to provide the best simulation environment for coupling GME to calculate the hydraulic force and allowing computing to be more efficient. In summary, this work can be easily extended to study other polygonal particles, and the results are useful for controlling the particulate movement in dynamic flow, especially in inertial microfluidics. It is beneficial to understand the complex motion of anisotropic particles; especially, the motion of free dendrite and the dendrite growth in dynamic environment [36] [37].

\section{Acknowledgements}

This work was supported by the National Natural Science Foundation of China (Grant Nos. 11862003, 11462003), the Key Project of Guangxi Natural Science Foundation (Grant No. 2017GXNSFDA198038), the Project of Guangxi Natural Science Foundation (Grant No. 2018JJA110023), the Innovation Project of Guangxi Graduate Education (Grant No. XYCSZ2019067), Guangxi "Bagui Scholar" Teams for Innovation and Research Project.

\section{Conflicts of Interest}

The authors declare no conflicts of interest regarding the publication of this paper.

\section{References}

[1] Kelso, R.M., Lim, T.T. and Perry, A.E. (1996) An Experimental Study of Round Jets in Cross-Flow. Journal of Fluid Mechanics, 306, 111-144. https://doi.org/10.1017/S0022112096001255

[2] Segre, G. and Silberberg, A. (1961) Radial Particle Displacements in Poiseuille Flow of Suspensions. Nature, 189, 209-201. https://doi.org/10.1038/189209a0

[3] Karnis, A., Goldsmith, H.L. and Mason, S.G. (1966) The Flow of Suspensions through Tubes: V. Inertial Effects. Canadian Journal of Chemical Engineering, 44, 181. https://doi.org/10.1002/cjce.5450440401

[4] Matas, J.P., Morris, J.F. and Guazzelli, E. (2004) Inertial Migration of Rigid Spherical Particles in Poiseuille Flow. Journal of Fluid Mechanics, 515, 171-195. https://doi.org/10.1017/S0022112004000254

[5] Huang, H.B., Yang, X. and Lu, X.Y. (2014) Sedimentation of an Ellipsoidal Particle in Narrow Tubes. Physics of Fluids, 26, Article ID: 053302.

https://doi.org/10.1063/1.4874606

[6] Di Carlo, D. (2009) Inertial Microfluidics. Lab on a Chip, 9, 3038-3046. https://doi.org/10.1039/b912547g

[7] Xiang, N., Chen, K. and Dai, Q. (2015) Inertia-Induced Focusing Dynamics of Microparticles throughout a Curved Microfluidic Channel. Nanofluids, 18, 29-39. https://doi.org/10.1007/s10404-014-1395-x 
[8] Liu, C., Xue, C. and Sun, J. (2016) A Generalized Formula for Inertial Lift on a Sphere in Microchannels. Lab on a Chip, 16, 884-892. https://doi.org/10.1039/C5LC01522G

[9] Ansumali, S. (2007) Hydrodynamics beyond Navier-Stokes: Exact Solution to the Lattice Boltzmann Hierarchy. Physical Review Letters, 98, Article ID: 124502. https://doi.org/10.1103/PhysRevLett.98.124502

[10] Qian, Y.H., d'Humières, D. and Lallemand, P. (1992) Lattice BGK Models for Navier-Stokes Equation. Europhysics Letters, 17, 479. https://doi.org/10.1209/0295-5075/17/6/001

[11] Sun, D.K., Wang, Y., Dong, A.P. and Sun, B.D. (2016) A Three-Dimensional Quantitative Study on the Hydrodynamic Focusing of Particles with the Immersed Boundary-Lattice Boltzmann Method. International Journal of Heat and Mass Transfer, 94, 306-315. https://doi.org/10.1016/j.ijheatmasstransfer.2015.11.012

[12] Wen, B., Chen, H., Qin, Z., He, B. and Zhang, C. (2016) Lateral Migration and Nonuniform Rotation of Suspended Ellipse in Poiseuille Flow. Computers \& Mathematics with Applications.

[13] Wen, B., Chen, Y., Zhang, R., Zhang, C. and Fang, H. (2013) Lateral Migration and Nonuniform Rotation of Biconcave Particle Suspended in Poiseuille Flow. Chinese Physics Letters, 30, Article ID: 064701. https://doi.org/10.1088/0256-307X/30/6/064701

[14] Huang, H.B., Yang, X., Krafczyk, M. and Lu, X.Y. (2012) Rotation of Spheroidal Particles in Couette Flows. Journal of Fluid Mechanics, 692, 369-394. https://doi.org/10.1017/jfm.2011.519

[15] Yang, X., Huang, H. and Lu, X. (2017) The Motion of a Neutrally Buoyant Ellipsoid Inside Square Tube Flows. Advances in Applied Mathematics and Mechanics, 9, 233-249. https://doi.org/10.4208/aamm.2015.m1376

[16] Laskovski, D., Stevenson, P. and Galvin, K.P. (2009) Lift and Drag Forces on an Isolated Cubic Particle in Pipe Flow. Chemical Engineering Research and Design, 87, 1573-1581. https://doi.org/10.1016/j.cherd.2009.05.002

[17] Bösch, F. and Karlin, I.V. (2013) Exact Lattice Boltzmann Equation. Physical Review Letters, 111, Article ID: 090601. https://doi.org/10.1103/PhysRevLett.111.090601

[18] Ansumali, S. and Karlin, I.V. (2002) Single Relaxation Time Model for Entropic Lattice Boltzmann Methods. Physical Review E, 65, Article ID: 056312. https://doi.org/10.1103/PhysRevE.65.056312

[19] Mazloomi, A.M., Chikatamarla, S.S. and Karlin, I.V. (2015) Entropic Lattice Boltzmann Method for Multiphase Flows. Physical Review Letters, 114, Article ID: 174502. https://doi.org/10.1103/PhysRevLett.114.174502

[20] Wen, B., Zhang, C. and Fang, H. (2017) Hydrodynamic Force Evaluation in Lattice Boltzmann Method. Science China Physics, Mechanics \& Astronomy, 47, Article ID: 070012. https://doi.org/10.1360/SSPMA2016-00404

[21] Lallemand, P. and Luo, L.S. (2003) Theory of the Lattice Boltzmann Method: Acoustic and Thermal Properties in Two and Three Dimensions. Physical Review E, 68, Article ID: 036706. https://doi.org/10.1103/PhysRevE.68.036706

[22] Bouzidi, M., Firdaouss, M. and Lallemand, P. (2001) Momentum Transfer of a Boltzmann-Lattice Fluid with Boundaries. Physics of Fluids, 13, 3452-3459. https://doi.org/10.1063/1.1399290

[23] Wang, J., Wang, D., Lallemand, P. and Luo, L.S. (2012) Lattice Boltzmann Simula- 
tions of Thermal Convective Flows in Two Dimensions. Computers \& Mathematics with Applications, 65, 262-286.

[24] Manacorda, A. and Puglisi, A. (2017) Lattice Model to Derive the Fluctuating Hydrodynamics of Active Particles with Inertia. Physical Review Letters, 119, Article ID: 208003. https://doi.org/10.1103/PhysRevLett.119.208003

[25] Ladd, A.J.C. (1994) Numerical Simulations of Particulate Suspensions via a Discretized Boltzmann Equation. Part 1. Theoretical Foundation. Journal of Fluid Mechanics, 271, 285-309. https://doi.org/10.1017/S0022112094001771

[26] Aidun, C.K., Lu, Y.N. and Ding, E.J. (2000) Direct Analysis of Particulate Suspensions with Inertia Using the Discrete Boltzmann Equation. Journal of Fluid Mechanics, 373, 287-311. https://doi.org/10.1017/S0022112098002493

[27] Chen, S.D., Pan, T.W. and Chang, C.C. (2012) The Motion of a Single and Multiple Neutrally Buoyant Elliptical Cylinders in Plane Poiseuille Flow. Physics of Fluids, 24, Article ID: 103302. https://doi.org/10.1063/1.4757387

[28] Zou, Q.S. and He, X.Y. (1997) On Pressure and Velocity Boundary Conditions for the Lattice Boltzmann BGK Model. Physics of Fluids, 9, 1591-1598.

https://doi.org/10.1063/1.869307

[29] Wen, B., Zhang, C., Tu, Y., Wang, C. and Fang, H. (2014) Galilean Invariant Fluid-Solid Interfacial Dynamics in Lattice Boltzmann Simulations. Journal of Computational Physics, 266, 161-170. https://doi.org/10.1016/j.jcp.2014.02.018

[30] Hu, H., Patankar, N. and Zhu, M. (2001) Direct Numerical Simulations of Fluid-Solid Systems Using the Arbitrary Lagrangian-Eulerian Technique. Journal of Computational Physics, 169, 427-462. https://doi.org/10.1006/jcph.2000.6592

[31] Huang, P., Hu, H. and Joseph, D. (1998) Direct Simulation of the Sedimentation of Elliptic Particles in Oldroyd-B Fluids. Journal of Fluid Mechanics, 362, 297-325. https://doi.org/10.1017/S0022112098008672

[32] Huang, P., Feng, J. and Joseph, D. (1994) The Turning Couples on an Elliptic Particle Settling in a Vertical Channel. Journal of Fluid Mechanics, 271, 1-16. https://doi.org/10.1017/S0022112094001667

[33] Velasco, A.E., Friedman, S.G., Pevarnik, M., Siwy, Z.S. and Taborek, P. (2012) Pressure-Driven Flow through a Single Nanopore. Physical Review E, 86, Article ID: 025302. https://doi.org/10.1103/PhysRevE.86.025302

[34] Yang, X., Huang, H. and Lu, X. (2015) Sedimentation of an Oblate Ellipsoid in Narrow Tubes. Physical Review E, 92, Article ID: 063009. https://doi.org/10.1103/PhysRevE.92.063009

[35] Wen, B., Li, H., Zhang, C. and Fang, H. (2012) Lattice-Type-Dependent Momentum-Exchange Method for Moving Boundaries. Physical Review E, 85, Article ID: 016704. https://doi.org/10.1103/PhysRevE.85.016704

[36] Sun, D., Wang, Y., Yu, H. and Han, Q. (2018) A Lattice Boltzmann Study on Dendritic Growth of a Binary Alloy in the Presence of Melt Convection. International Journal of Heat and Mass Transfer, 123, 213-226. https://doi.org/10.1016/j.ijheatmasstransfer.2018.02.053

[37] Sun, D., Pan, S., Han, Q. and Sun, B. (2016) Numerical Simulation of Dendritic Growth in Directional Solidification of Binary Alloys Using a Lattice Boltzmann Scheme. International Journal of Heat and Mass Transfer, 103, 821-831. https://doi.org/10.1016/j.ijheatmasstransfer.2016.07.055 


\section{Nomenclature}

$\rho:$ Density of water $\left[\mathrm{kg} / \mathrm{m}^{3}\right]$

$x$ : Lattice space

$t$. Time (dimensionless)

$f_{i}$ : Distribution function along $i$ direction

$\boldsymbol{u}$ : Macroscopic velocity vector $[\mathrm{m} / \mathrm{s}]$

$\boldsymbol{e}_{i}$ : Unit velocity vector along $i$ direction

$q$ : Non-dimensional fraction

$\boldsymbol{u}_{w}$ : Velocity of the moving boundary $[\mathrm{m} / \mathrm{s}]$

$\tau$ : Dimensionless time

$F$ : Force $[\mathrm{N}]$

$T$ : Torque $[\mathrm{N} \cdot \mathrm{m}]$

$R$ : Centroid position

$\boldsymbol{v}$. Kinematic viscosity coefficient $\left[\mathrm{m}^{2} / \mathrm{s}\right]$

Re: Reynolds number (dimensionless)

$H$ : The width of the channel [m]

$U$ : The mean fluid velocity in Poiseuille flow without a particle $[\mathrm{m} / \mathrm{s}]$

T: Period

$Y$ : Vertical axis of the pipe $[\mathrm{mm}]$

$\omega:$ Rotation velocity $[\mathrm{rad} / \mathrm{s}]$

$\delta t:$ Lattice step time (dimensionless) 